\title{
AN INSTRUMENT IN HYPERBOLIC GEOMETRY
}

\author{
M. W. AL-DHAHIR ${ }^{1}$
}

In addition to straight edge and compasses, the classical instruments of Euclidean geometry, we have in hyperbolic geometry the horocompass and the hypercompass. By a straight edge, or ruler, we draw the line joining any two distinct points, and by the compasses we construct a circle with given center and radius. The horocompass is used to draw a horocycle through a given point when its diameter through the point with its direction are given. If the central line and radius of a hypercycle are given, we can draw it by the hypercompass.

Although ruler and compasses have been generally used in the solutions of construction problems in hyperbolic geometry $[1 ; 2$, p. 191 , pp. $204-206 ; 3$, p. 394 ], other instruments have been introduced, and the relationships among these instruments, together with some restrictions, have been studied in recent years [2, pp. 289-291]. An important result in this connection is the following theorem [2, p. 290].

THEOREM A. In conjunction with a ruler, the three compasses are equivalent.

Recently [3, p. 389], a different geometrical tool, called the parallel-ruler, has been considered. For any point $A$ and any ray $a$, not incident to $A$, we can draw, with this ruler, a line through $A$ parallel to $a$. As in Euclidean geometry, the parallel-ruler may also be used as an ordinary ruler. Hence the following result has been obtained [3].

TheOREM B. Any construction that can be performed by a ruler and compasses can be performed by a parallel-ruler.

The purpose of the present note is to introduce a new instrument which may be described as follows. For every pair of distinct lines, a line can be drawn parallel to either and perpendicular to the other. When the two given lines subtend a right angle, we agree to consider each one to be parallel to itself and perpendicular to the other. Like the parallel-ruler, we shall also use this "parallel-perpendicular ruler" as an ordinary ruler. Because it is characteristic of hyperbolic geometry (unlike the parallel-ruler which may also be used in Euclidean

Presented to the Society, April 14, 1961; received by the editors February 6, 1961.

1 Fellow of the National Research Council of Canada. 
geometry) we call it simply the hyperbolic ruler. Our aim is to establish the following main result.

TheORem C. Any hyperbolic construction that can be performed by a ruler and any one of the three compasses can be performed by the hyperbolic ruler.

We shall give, in what follows, a sequence of constructions using the hyperbolic ruler alone. By these constructions we prove that any construction that can be obtained by a parallel-ruler, can be obtained by a hyperbolic ruler; and then, by Theorems A and B, Theorem C follows.

(i) The common perpendicular to two ultraparallel lines.

Let $a, a^{\prime}$ be two ultraparallel lines. Draw $A I \perp a \& \| a^{\prime}$, and $A^{\prime} I^{\prime} \perp a \& \| a^{\prime}$ (in the other direction). Also, draw $B I^{\prime} \perp A I \& \| a^{\prime}$ and $B^{\prime} I \perp A^{\prime} I^{\prime} \& \| a^{\prime}$. If $B I^{\prime} \cdot B^{\prime} I=O, A B^{\prime} \cdot A^{\prime} B=O^{\prime}$, then $O O^{\prime}$ is the common perpendicular to $a$ and $a^{\prime}$ (Figure 1).

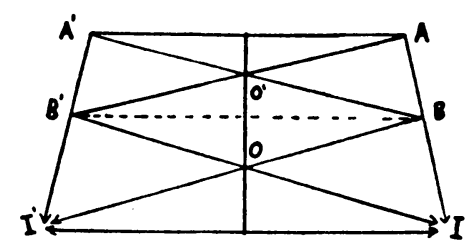

FIGURE 1

To prove this, we have $\Varangle B O I=\pi(O B)=\Varangle B^{\prime} O I^{\prime}=\pi\left(O B^{\prime}\right)$, yielding $O B=O B^{\prime}$. The figure $A B B^{\prime} A^{\prime}$ is an equiangular birectangle and therefore $A B=A^{\prime} B^{\prime}$. Since the two diagonals of the isosceles birectangle make equal angles with its bases, we may reflect in the line $O O^{\prime}$ and obtain the result.

(ii) The angle bisector.

Let $\Varangle I A I^{\prime}$ be given. Assuming $\Varangle I A I^{\prime} \neq \pi / 2$, we have two cases to be discussed.

( $\alpha$ ) If $\varangle I A I^{\prime}<\pi / 2$, draw $B I^{\prime} \perp A I \& \| A I^{\prime}$ and $B^{\prime} I \perp A I^{\prime} \& \| A I$. Denoting the point $B I^{\prime} \cdot B^{\prime} I$ by $C$, we obtain $\pi(B C)=\pi\left(B^{\prime} C\right)$ and thus $B C=B^{\prime} C$. Similarly, $A B=A B^{\prime}$ and hence $A C$ bisects the given angle.

$(\beta)$ Let $\Varangle I A I^{\prime}>\pi / 2$. Draw $B I^{\prime}$ and $B^{\prime} I$ as in $(\alpha),\left(\pi-\Varangle I A I^{\prime}\right.$ $\varangle \pi / 2)$; hence $A B=A B^{\prime}$. Now three cases present themselves:

$\left(\beta_{1}\right)$ If $I B^{\prime}$ intersects $I^{\prime} B$, say in $A^{\prime}$, then it is obvious that $A A^{\prime}$ is the bisector.

$\left(\beta_{2}\right)$ Assume $I B^{\prime} \| I^{\prime} B$. Join $B B^{\prime}$ and draw $O I^{\prime \prime} \perp B B^{\prime} \& \| B^{\prime} I^{\prime \prime}$. As $\Varangle O B I^{\prime \prime}=\Varangle O B^{\prime} I^{\prime \prime}$, we have $O B=O B^{\prime}$. Hence $I^{\prime \prime} O \perp B B^{\prime}$ at its mid- 
point and consequently $O A$ bisects $\Varangle I A I^{\prime}$ (Figure 2).

$\left(\beta_{3}\right)$ Finally suppose $I B^{\prime}$ is ultraparallel to $I^{\prime} B$. Construct their common perpendicular $C C^{\prime}$ (i). If $C B^{\prime} \cdot B C^{\prime}=D$ then $A D$ is the angle bisector. Indeed, since $A B=A B^{\prime}$, we have $\Varangle A B B^{\prime}=\Varangle A B^{\prime} B$ which implies $B C^{\prime}=B^{\prime} C$ and $B D=B^{\prime} D$. Now it follows easily that $\Varangle B A O$ $=\varangle B^{\prime} A O$ (Figure 3 ).

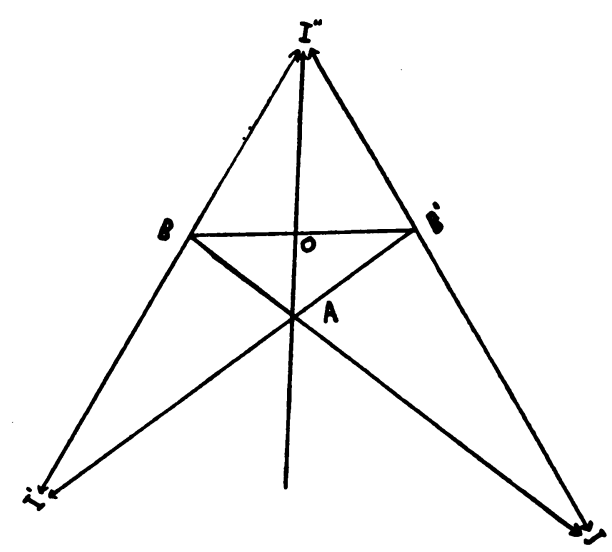

FIGURE 2

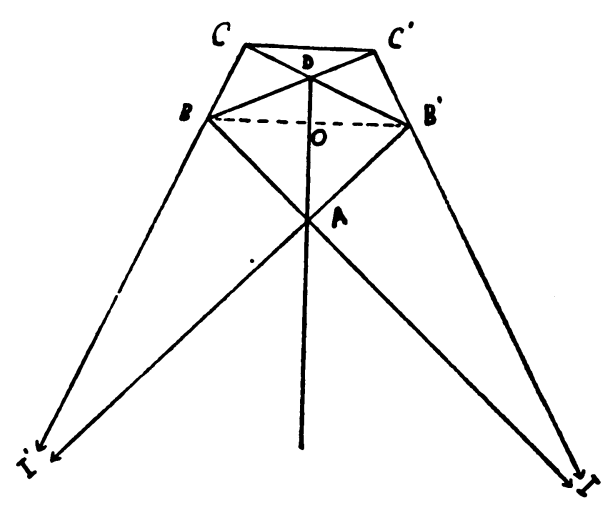

FIGURE 3

(iii) The common parallel to two lines.

If $a$ and $a^{\prime}$ be any two distinct lines, we consider three cases.

( $\alpha$ ) Let $a$ intersect $a^{\prime}$ in $O$. Bisect any one of the two resulting adjacent angles (ii), and draw a line perpendicular to the angle bisector and parallel to (one of them, say) $a$. Clearly this line is also parallel to $a^{\prime}$. When $a$ is not perpendicular to $a^{\prime}$, this construction provides four common parallels corresponding to the four pairs of 
rays resulting from the two given intersecting lines. The case when $a$ is perpendicular to $a^{\prime}$ remains to be treated in Remark 1 following construction (vi).

( $\beta$ ) Suppose $a \| a^{\prime}$. Let $A I^{\prime} \perp a \& \| a^{\prime}$, and $A^{\prime} I \perp a^{\prime} \& \| a$. Denote the point $A I^{\prime} \cdot A^{\prime} I$ by $B$. As $\varangle I B I^{\prime} \neq \pi / 2$ (in fact $>\pi / 2$ ) the case reduces to $(\alpha)$ and the common parallel to the two rays $B I$ and $B I^{\prime}$ (see Figure 4) may be constructed.

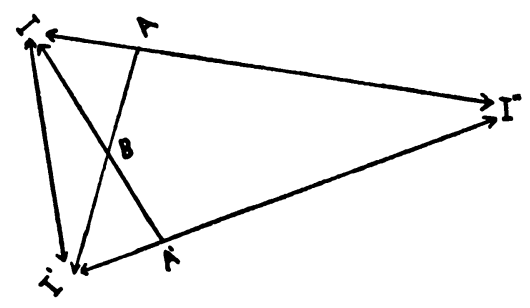

FIGURE 4

( $\gamma$ ) If $a$ is ultraparallel to $a^{\prime}$, draw $B I^{\prime} \perp a \& \| a^{\prime}$ and $B^{\prime} I \perp a^{\prime} \& \| a$, where $B I^{\prime}$ and $B^{\prime} I$ are taken on the same side of the common perpendicular to $a, a^{\prime}$. Hence we may denote $B I^{\prime} \cdot B^{\prime} I$ by $C$ and obtain $\varangle I C I^{\prime} \neq \pi / 2$; and thus the rest follows as in the previous cases. We note that in this case we have two common parallels, each lying in a side of the common perpendicular.

(iv) The perpendicular to a line at a point.

Let $a$ be a given line with a given point $A$ on it. We shall erect the perpendicular to $a$ at $A$. Let $a^{\prime} \neq a$ be an arbitrary line through $A$. If $a^{\prime} \perp a$ we have the result; otherwise, draw $B I \perp a \& \| a^{\prime}$ and $C I^{\prime} \perp a$ $\& \| a^{\prime}$ (in the other direction). Construct the common parallel $I I^{\prime \prime}$ to $B I$ and $I^{\prime} C$, (iii, $\beta$ ), (see Figure 5). As $I I^{\prime \prime}$ is ultraparallel to $a$, we may construct their common perpendicular (i), which will be the line of symmetry for the doubly asymptotic birectangle $I B C I^{\prime \prime}$, and therefore it must pass through the point $A$ which is the midpoint of its lower base $B C$.

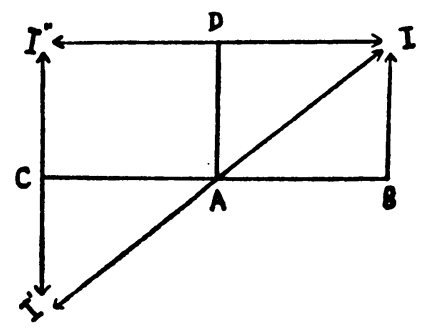

FIGURE 5 
(v) The midpoint of a line segment.

For a given segment $A B$, construct $A I$ and $B I^{\prime}$ perpendicular to $A B$ (iv), and then construct the common parallel $I I^{\prime}$ (iii, $\gamma$ ). Since $I I^{\prime}$ is ultraparallel to $A B$, construct their common perpendicular $C C^{\prime}$ (i). Clearly $C$ is the midpoint of $A B$, which may be seen by reflecting in the line $C C^{\prime}$.

(vi) Given a point $A$ on one arm of an angle $I O I^{\prime}$, to construct a point $B$ on the other arm such that $O A=O B$.

We consider three cases as follows.

( $\alpha$ ) Let $\Varangle I O I^{\prime}<\pi / 2$. Draw $A^{\prime} I^{\prime} \perp O I \& \| O I^{\prime}$ and $B^{\prime} I \perp O I^{\prime} \& \| O I$. By (ii, $\alpha$ ) the point $A^{\prime} I^{\prime} \cdot B^{\prime} I=C$ lies on the bisector. If we denote $A B^{\prime} \cdot O C$ by $C^{\prime}$, then it is easily seen that $A^{\prime} C^{\prime}$ intersects $O I^{\prime}$ in the desired point $B$.

( $\beta$ ) Let $\Varangle I O I^{\prime}>\pi / 2$. Bisect it by the line $O I^{\prime \prime}$ (ii, $\beta$ ). Applying $(\alpha)$ to the two acute angles $I O I^{\prime \prime}$ and $I^{\prime \prime} O I^{\prime}$, we find $C$ on $O I^{\prime \prime}$ and $B$ on $O I$ such that $O A=O C=O B$.

( $\gamma$ ) If $\Varangle I O I^{\prime}=\pi / 2$, let $O I^{\prime \prime}$ be any line through it. Now the two angles $I O I^{\prime \prime}$ and $I^{\prime \prime} O I^{\prime}$ are each less than $\pi / 2$ and hence by twice application of $(\alpha)$ we obtain the result.

REMARK 1. It is worth noting that the last case of the preceding construction provides solutions for the right angle cases which were avoided in (ii) and (iii).

(ii, $\gamma$ ). If $\Varangle I A I^{\prime}=\pi / 2$, let $B \neq A$ be any point on $A I$. We find a point $B^{\prime}$, on $A I^{\prime}$, such that $A B^{\prime}=A B$ (vi, $\gamma$ ). Bisecting $B B^{\prime}$ by the point $C(\mathrm{v})$, we obtain $A C$ the required bisector of $\Varangle I A I^{\prime}$.

(iii, $\alpha^{\prime}$ ). In construction (iii, $\alpha$ ), let $a \perp a^{\prime}$. Bisect their right angle by the line $O I^{\prime \prime}$ (ii, $\gamma$ ) and draw $B I^{\prime} \perp O I^{\prime \prime} \& \| O I^{\prime}$. Because $I^{\prime} B$ is parallel to $O I$, the line $I I^{\prime}$ is the common parallel to $a$ and $a^{\prime}$ (in the two designated senses). There are four such lines corresponding to the four pairs of rays of the two intersecting lines $a, a^{\prime}$.

REMARK 2. The point $B$ in the above construction (vi), is said to correspond to the point $A$ in the sense of Gauss [2, p. 215]. By this construction we may obtain a set of corresponding points on a pencil of intersecting lines using hyperbolic ruler only. The method may be extended without difficulty to the parallel and ultraparallel pencils.

(vii) Given a segment $O A$ on $a$, to construct a point $B \neq A$, on $a$, such that $O B=O A$.

Let $a^{\prime} \neq a$ be an arbitrary line through $O$. Using (vi), construct $B^{\prime}$ on $a^{\prime}$ and $B$ on $a$ so that $O B^{\prime}=O A=O B$.

(viii) Given $\nless I O I^{\prime} \neq \pi / 2$, to construct a ray $O I^{\prime \prime}$ such that $\nless I O I^{\prime \prime}$ $=\varangle I O I^{\prime}$. 
Draw $B I^{\prime} \perp O I \& \| O I^{\prime}$ (if $\Varangle I O I^{\prime}>\pi / 2$ produce $I^{\prime} O$ ). At any point $C$ on the segment $O B$, construct $C C^{\prime} \perp O I$ (iv). If $C C^{\prime}$ intersects the line $O I^{\prime}$ at $C^{\prime}$, find $C^{\prime \prime}$, on $C C^{\prime}$, such that $C C^{\prime \prime}=C C^{\prime}$ (vii) and join $O C^{\prime \prime}$. Since $C^{\prime \prime}$ is the symmetric point of $C^{\prime}$ with respect to $O I$ then $O C^{\prime \prime}$ is the required ray $O I^{\prime \prime}$.

(ix) The perpendicular to a line from an outside point.

Let $a$ be a given line and $P$ a point outside $a$. Draw any line $a^{\prime}$ through $P$ intersecting $a$ in some point $O$. If $P O$ is not perpendicular to $a$, draw a ray $a^{\prime \prime}$ through $O$ making an angle with $a$ equal to the angle $a^{\prime}$ makes with $a$ (viii). Construct $P^{\prime \prime}$ on $a^{\prime \prime}$ such that $O P^{\prime \prime}$ $=O P$ (vi). Since $P^{\prime \prime}$ is the symmetric point of $P$ with respect to $a$, then $P Q \perp a$ where $Q=P P^{\prime \prime} \cdot a$.

(x) Given a line $a, a$ segment $A B$ and a point $A^{\prime}$ both on $a$, to find $a$ point $B^{\prime}$ on a such that $A^{\prime} B^{\prime}=A B$.

Bisect $B A^{\prime}$ at $O(\mathrm{v})$ and construct $A I$ and $O I^{\prime}$ both perpendicular to $a$ (iv). From any point $C$ on $A I$, construct $C O^{\prime} \perp O I^{\prime}$ (ix) and extend $C O^{\prime}$ to $C^{\prime}$ such that $O^{\prime} C^{\prime}=C O^{\prime}$ (vii). Draw $C^{\prime} B^{\prime} \perp a$ (ix). Inspection of the figure $A C C^{\prime} B^{\prime}$ reveals its symmetry with respect to $O O^{\prime}$. Hence $O A=O B^{\prime}$ and consequently $A^{\prime} B^{\prime}=A B$.

(xi) The parallel line to a ray.

Now let $a$ be a given line and $P$ a given point outside $a$. Draw $P Q \perp a$ (ix) and $P I \perp P Q$ (iv). From any point $R \neq Q$, on $a$, construct $R S \perp P I$ (ix). As $\Varangle Q R S<\pi / 2$, draw $T I^{\prime} \perp S R \& \| a$. Find a point $T^{\prime}$ on $S T$, such that $S T^{\prime}=R T(\mathbf{x})$. Then the line $P T^{\prime}$ is the (right) parallel to $a$; (the left parallel to $a$ may be constructed similarly on the other side of $P Q$ ).

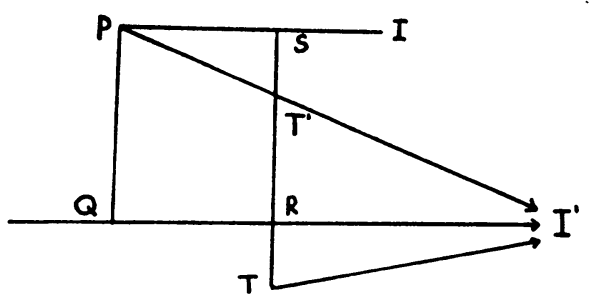

Figure 6

To prove this we observe that the right-angled triangle $P S T^{\prime}$ is associated with the trirectangle $P Q R S$ in the Engel chain of alternate triangles and trirectangles $\left[2\right.$, p. 279]. Hence $P T^{\prime}=Q R$ and consequently $P T^{\prime} \| a$. 


\section{REFERENCES}

1. M. W. Al-Dhahir and R. N. Shekoury, Constructions in the hyperbolic plane, Proc. Iraqi Sci. Soc. 2 (1958), 1-6.

2. H. S. M. Coxeter, Non-euclidean geometry, 3d ed., Univ. of Toronto Press, 1957.

3. F. Handest, Constructions in hyperbolic geometry, Canad. J. Math. 8 (1956), 389-394.

UNIVERSITY OF BAGHDAD AND

UNIVERSITY OF TORONTO

\section{ON THE EULER CHARACTERISTIC OF POLYHEDRA}

\section{CHARLES E. WATTS ${ }^{1}$}

1. The principal object of this note is to give an extremely simple axiomatic characterization of the Euler characteristic for finite polyhedra. The idea of the construction used was suggested by the definition of the Grothendieck group of sheaves over a space [1]. Unless otherwise noted, "space" always means "triangulable space with base point," and a "pair" is a "triangulable pair of spaces with common base point." Mappings of spaces always preserve base points. If $(X, A)$ is a pair, then $X / A$ denotes the space obtained from $X$ by identifying $A$ to a point.

2. Let $F$ be the free abelian group generated by all homeomorphism classes of spaces. We denote by $N$ the subgroup of $F$ generated by all elements of the form

$$
X-A-X / A \text {, }
$$

where $(X, A)$ is a pair. Set $\Gamma=F / N$ and let $\gamma: F \rightarrow \Gamma$ be the natural homomorphism.

Lemma 1. If $X, Y$ are spaces, then $\gamma(X \vee Y)=\gamma X+\gamma Y$.

The proof is obvious.

Lemma 2. If $E^{n}$ is an $n$-cell (with base point on the boundary) then $\gamma E^{n}=0$.

Received by the editors February 27, 1961.

1 This work was done while the author was a National Science Foundation Postdoctoral Fellow. 